\title{
SOME INTEGRAL INEQUALITIES OF HERMITE-HADAMARD TYPE FOR s-GEOMETRICALLY CONVEX FUNCTIONS
}

\author{
HONG-PING YIN, JING-YU WANG, AND FENG QI \\ Received 19 November, 2017
}

\begin{abstract}
In the paper, the authors present some integral inequalities of the Hermite-Hadamard type for $s$-geometrically convex functions and for the product of two $s$-geometrically convex functions.
\end{abstract}

2010 Mathematics Subject Classification: 26D15; 26A51; 26D20; 41A55

Keywords: integral inequality, Hermite-Hadamard type, $s$-geometrically convex function, product

\section{INTRODUCTION}

We recall the definitions of classical convex functions and geometrically convex functions.

Definition 1. Let $f: I \subseteq \mathbb{R}=(-\infty, \infty) \rightarrow \mathbb{R}$. If the inequality

$$
f(\lambda x+(1-\lambda) y) \leq \lambda f(x)+(1-\lambda) f(y)
$$

is valid for all $x, y \in I$ and $\lambda \in[0,1]$, then $f$ is called the convex function on $I$; if the inequality (1.1) reverses, then $f$ is called the concave function on $I$.

Definition 2. Let $f: I \subseteq \mathbb{R}_{+}=(0, \infty) \rightarrow \mathbb{R}_{+}$. If the inequality

$$
f\left(x^{\lambda} y^{1-\lambda}\right) \leq f^{\lambda}(x) f^{1-\lambda}(y)
$$

is sound for any $x, y \in I$ and $\lambda \in[0,1]$, then $f$ is called the geometrically convex function; if the inequality of (1.2) reverses, then $f$ is called the geometrically concave function.

The concept of classical convex functions has been generalized or extended widely in recent decades. Some of them can be reformulated as follows.

Definition 3 ([3,5]). Let $f: I \subseteq \mathbb{R} \rightarrow \mathbb{R}_{0}=[0, \infty)$ and $s \in(0,1]$. If the inequality

$$
f(\lambda x+(1-\lambda) y) \leq \lambda^{s} f(x)+(1-\lambda)^{s} f(y)
$$

holds for all $x, y \in I$ and $\lambda \in[0,1]$, then $f$ is called the $s$-convex function on $I$.

(c) 2018 Miskolc University Press 
Definition 4 ([14,15]). Let $s \in(0,1]$ and $f: I \subseteq R_{+} \rightarrow R_{+}$. If the inequality

$$
f\left(x^{\lambda} y^{1-\lambda}\right) \leq f^{\lambda^{s}}(x) f^{(1-\lambda)^{s}}(y)
$$

validates for any $x, y \in I$ and $\lambda \in[0,1]$, then $f$ is called the $s$-geometrically convex function on $I$.

For classical convex functions, we have the famous Hermite-Hadamard integral inequality below.

Theorem 1. Let $f:[a, b] \subset \mathbb{R} \rightarrow \mathbb{R}$ be a convex function on $[a, b]$. Then

$$
f\left(\frac{a+b}{2}\right) \leq \frac{1}{b-a} \int_{a}^{b} f(x) \mathrm{d} x \leq \frac{f(a)+f(b)}{2} .
$$

If $f$ is a concave function on I, then the inequality (1.3) reverses.

In the literature, there have existed some integral inequalities of the HermiteHadamard type on classical convex functions and $s$-convex functions. Some of them can be reformulated as follows.

Theorem 2 ([4, Theorem 2.2]). Let $f: I^{\circ} \subseteq \mathbb{R} \rightarrow \mathbb{R}$ be a differentiable mapping and $a, b \in I^{\circ}$ with $a<b$. If $\left|f^{\prime}\right|$ is convex on $[a, b]$, then

$$
\left|\frac{f(a)+f(b)}{2}-\frac{1}{b-a} \int_{a}^{b} f(x) \mathrm{d} x\right| \leq \frac{(b-a)}{8}\left(\left|f^{\prime}(a)\right|+\left|f^{\prime}(b)\right|\right) .
$$

Theorem 3 ([7, Theorems 2.3 and 2.4]). Let $f: I \subseteq \mathbb{R}_{0} \rightarrow \mathbb{R}$ be differentiable on $I^{\circ}$ and $a, b \in I$ with $a<b$. If $\left|f^{\prime}\right|^{p}$ is s-convex on $[a, b]$ for some $s \in(0,1]$ and $p>1$, then

$$
\left|f\left(\frac{a+b}{2}\right)-\frac{1}{b-a} \int_{a}^{b} f(x) \mathrm{d} x\right| \leq \frac{b-a}{16}\left(\frac{4}{p+1}\right)^{1 / p}\left(\left|f^{\prime}(a)\right|+\left|f^{\prime}(b)\right|\right)
$$

and

$$
\begin{aligned}
&\left|f\left(\frac{a+b}{2}\right)-\frac{1}{b-a} \int_{a}^{b} f(x) \mathrm{d} x\right| \leq \frac{b-a}{4}\left(\frac{4}{p+1}\right)^{1 / p}\left\{\left[\left|f^{\prime}(a)\right|^{p /(p-1)}\right.\right. \\
&\left.\left.+3\left|f^{\prime}(b)\right|^{p /(p-1)}\right]^{1-1 / p}+\left[3\left|f^{\prime}(a)\right|^{p /(p-1)}+\left|f^{\prime}(b)\right|^{p /(p-1)}\right]^{1-1 / p}\right\} .
\end{aligned}
$$

Theorem 4 ([6, Theorem 3]). Let $f: I \subseteq \mathbb{R}_{0} \rightarrow \mathbb{R}$ be differentiable on $I^{\circ}, a, b \in I$ with $a<b$, and $f^{\prime} \in L[a, b]$. If $\left|f^{\prime}\right|^{q}$ is $s$-convex on $[a, b]$ for some $s \in(0,1]$ and $q>1$, then

$$
\begin{aligned}
& \left|\frac{f(a)+f(b)}{2}-\frac{1}{b-a} \int_{a}^{b} f(x) \mathrm{d} x\right| \leq \frac{b-a}{2}\left[\frac{q-1}{2(2 q-1)}\right]^{1 / p}\left(\frac{1}{s+1}\right)^{1 / q} \\
& \quad \times\left\{\left[\left|f^{\prime}(a)\right|^{q}+\left|f^{\prime}\left(\frac{a+b}{2}\right)\right|^{q}\right]^{1 / q}+\left[\left|f^{\prime}(b)\right|^{q}+\left|f^{\prime}\left(\frac{a+b}{2}\right)\right|^{q}\right]^{1 / q}\right\} .
\end{aligned}
$$


In recent decades, many new integral inequalities of the Hermite-Hadamrd type for diverse new kinds of convex functions have been created and established. For detailed information, please refer to [1-4,6-15] and closely related references therein.

In this paper, we will establish some new integral inequalities of the HermiteHadamard type for $s$-geometrically convex functions.

\section{SOME NEW INTEGRAL INEQUALITIES}

We now start out to establish some integral inequalities of the Hermite-Hadamard type for $s$-geometrically convex functions.

Theorem 5. Let $f: I \subseteq \mathbb{R}_{+} \rightarrow \mathbb{R}_{+}$be an integrable function, $a, b \in I$ with $a<b$, and $s \in(0,1]$. If $f$ is an $s$-geometrically convex function, then

$$
f^{(1 / 2)^{1-s}}(\sqrt{a b}) \leq \frac{1}{\ln b-\ln a} \int_{a}^{b} \frac{f(x)}{x} \mathrm{~d} x \leq[f(a) f(b)]^{1-s} L\left(f^{s}(a), f^{s}(b)\right),
$$

where the logarithmic mean $L(u, v)$ is defined by

$$
L(u, v)= \begin{cases}\frac{v-u}{\ln v-\ln u}, & u \neq v ; \\ u, & u=v .\end{cases}
$$

Proof. By changing the variable $x=a^{t} b^{1-t}$ for $t \in[0,1]$ and by the $s$-geometric convexity, we have

$$
\frac{1}{\ln b-\ln a} \int_{a}^{b} \frac{f(x)}{x} \mathrm{~d} x=\int_{0}^{1} f\left(a^{t} b^{1-t}\right) \mathrm{d} t \leq \int_{0}^{1} f^{t^{s}}(a) f^{(1-t)^{s}}(b) \mathrm{d} t .
$$

In [2], it was obtained that the inequality

$$
\eta^{t^{s}} \leq \eta^{s t+1-s}
$$

is valid for $\eta \geq 1,0 \leq t \leq 1$, and $0<s \leq 1$. When $s \in(0,1)$, the $s$-geometrically convex function satisfies $f(x) \geq 1$. Consequently, since $f(a), f(b) \geq 1$, we arrive at

$$
\begin{aligned}
\int_{0}^{1} f^{t^{s}}(a) f^{(1-t)^{s}}(b) \mathrm{d} t & \leq \int_{0}^{1} f^{s t+1-s}(a) f^{s(1-t)+1-s}(b) \mathrm{d} t \\
& =[f(a) f(b)]^{1-s} L\left(f^{s}(a), f^{s}(b)\right) .
\end{aligned}
$$

Due to $\sqrt{a b}=\sqrt{a^{t} b^{1-t} b^{t} a^{1-t}}$ for all $t \in[0,1]$, by the $s$-geometric convexity, we find

$$
f^{(1 / 2)^{1-s}}(\sqrt{a b}) \leq\left[f\left(a^{t} b^{1-t}\right) f\left(b^{t} a^{1-t}\right)\right]^{1 / 2} \leq \frac{f\left(a^{t} b^{1-t}\right)+f\left(b^{t} a^{1-t}\right)}{2} .
$$

Integrating with respect to $t \in[0,1]$ on both sides of the above inequality gains

$$
f^{(1 / 2)^{1-s}}(\sqrt{a b}) \leq \frac{1}{2} \int_{0}^{1}\left[f\left(a^{t} b^{1-t}\right)+f\left(b^{t} a^{1-t}\right)\right] \mathrm{d} t=\frac{1}{\ln b-\ln a} \int_{a}^{b} \frac{f(x)}{x} \mathrm{~d} x .
$$


The proof of Theorem 5 is complete.

Theorem 6. Let $f: I \subseteq \mathbb{R}_{+} \rightarrow \mathbb{R}_{+}$be an integrable function, $a, b \in I$ with $a<b$, and $s \in(0,1]$. If $f$ is an $s$-geometrically convex function on $[a, b]$, then

$\sqrt{a b} f^{(1 / 2)^{1-s}}(\sqrt{a b}) \leq \frac{1}{\ln b-\ln a} \int_{a}^{b} f(x) \mathrm{d} x \leq[f(a) f(b)]^{1-s} L\left(a f^{s}(a), b f^{s}(b)\right)$.

where $L(u, v)$ is the logarithmic mean defined by (2.1).

Proof. Taking $x=a^{t} b^{1-t}$ for $t \in[0,1]$, using Definition 4, and employing the inequality (2.2) lead to

$$
\begin{aligned}
\frac{1}{\ln b-\ln a} \int_{a}^{b} f(x) \mathrm{d} x & =\int_{0}^{1} a^{t} b^{1-t} f\left(a^{t} b^{1-t}\right) \mathrm{d} t \leq \int_{0}^{1} a^{t} b^{1-t} f^{t^{s}}(a) f^{(1-t)^{s}}(b) \mathrm{d} t \\
& \leq \int_{0}^{1} a^{t} b^{1-t} f^{s t+1-s}(a) f^{s(1-t)+1-s}(b) \mathrm{d} t \\
& =[f(a) f(b)]^{1-s} L\left(a f^{s}(a), b f^{s}(b)\right)
\end{aligned}
$$

and

$$
\begin{aligned}
\sqrt{a b} f^{(1 / 2)^{1-s}}(\sqrt{a b}) & =\int_{0}^{1} \sqrt{a^{t} b^{1-t} b^{t} a^{1-t}} f^{(1 / 2)^{1-s}}\left(\sqrt{a^{t} b^{1-t} b^{t} a^{1-t}}\right) \mathrm{d} t \\
& \leq \int_{0}^{1} \sqrt{a^{t} b^{1-t} b^{t} a^{1-t}}\left[f\left(a^{t} b^{1-t}\right) f\left(b^{t} a^{1-t}\right)\right]^{1 / 2} \mathrm{~d} t \\
& \leq \frac{1}{2} \int_{0}^{1}\left[a^{t} b^{t} f\left(a^{t} b^{1-t}\right)+b^{t} a^{1-t} f\left(b^{t} a^{1-t}\right)\right] \mathrm{d} t \\
& =\frac{1}{\ln b-\ln a} \int_{a}^{b} f(x) \mathrm{d} x .
\end{aligned}
$$

The proof of Theorem 6 is complete.

Theorem 7. Let $f, g: I \subseteq \mathbb{R}_{+} \rightarrow \mathbb{R}_{+}$be integrable functions, $a, b \in I$ with $a<$ $b$, and $s_{1}, s_{2} \in(0,1]$. If $f$ is an $s_{1}$-geometrically convex function and $g$ is an $s_{2}$ geometrically convex function on $[a, b]$, then

$$
\begin{aligned}
f^{(1 / 2)^{1-s_{1}}}(\sqrt{a b}) g^{(1 / 2)^{1-s_{2}}}(\sqrt{a b}) \leq \frac{1}{\ln b-\ln a} \int_{a}^{b} \frac{f(x) g(x)}{x} \mathrm{~d} x \\
\leq[f(a) f(b)]^{1-s_{1}}[g(a) g(b)]^{1-s_{2}} L\left(f^{s_{1}}(a) g^{s_{2}}(a), f^{s_{1}}(b) g^{s_{2}}(b)\right),
\end{aligned}
$$

where $L(u, v)$ is the logarithmic mean defined by (2.1).

Proof. Letting $x=a^{t} b^{1-t}$ for $t \in[0,1]$, using the $s$-geometric convexity, and utilizing the inequality (2.2) result in

$$
\frac{1}{\ln b-\ln a} \int_{a}^{b} \frac{f(x) g(x)}{x} \mathrm{~d} x=\int_{0}^{1} f\left(a^{t} b^{1-t}\right) g\left(a^{t} b^{1-t}\right) \mathrm{d} t
$$




$$
\begin{gathered}
\leq \int_{0}^{1} f^{t^{s_{1}}}(a) f^{(1-t)^{s_{1}}}(b) g^{t^{s_{1}}}(a) g^{(1-t)^{s_{2}}}(b) \mathrm{d} t \\
\leq \int_{0}^{1} f^{s_{1} t+1-s_{1}}(a) f^{s_{2}(1-t)+1-s_{2}}(b) g^{s_{1} t+1-s_{1}}(a) g^{s_{2}(1-t)+1-s_{2}}(b) \mathrm{d} t \\
=[f(a) f(b)]^{1-s_{1}}[g(a) g(b)]^{1-s_{2}} L\left(f^{s_{1}}(a) g^{s_{2}}(a), f^{s_{1}}(b) g^{s_{2}}(b)\right) .
\end{gathered}
$$

For $t \in[0,1]$, we have

$$
\begin{aligned}
& f^{(1 / 2)^{1-s_{1}}}(\sqrt{a b}) g^{(1 / 2)^{1-s_{2}}}(\sqrt{a b}) \\
& =f^{(1 / 2)^{1-s_{1}}}\left(\sqrt{a^{t} b^{1-t} b^{t} a^{1-t}}\right) g^{(1 / 2)^{1-s_{2}}}\left(\sqrt{a^{t} b^{1-t} b^{t} a^{1-t}}\right) \\
& \leq\left[f\left(a^{t} b^{1-t}\right) f\left(b^{t} a^{1-t}\right) g\left(a^{t} b^{1-t}\right) g\left(b^{t} a^{1-t}\right)\right]^{1 / 2} \\
& \leq \frac{f\left(a^{t} b^{1-t}\right) g\left(a^{t} b^{1-t}\right)+f\left(b^{t} a^{1-t}\right) g\left(b^{t} a^{1-t}\right)}{2} \text {. }
\end{aligned}
$$

Integrating on both sides with respect to $t \in[0,1]$ leads to

$$
\begin{aligned}
& f^{(1 / 2)^{1-s_{1}}}(\sqrt{a b}) g^{(1 / 2)^{1-s_{2}}}(\sqrt{a b}) \\
\leq & \frac{1}{2} \int_{0}^{1}\left[f\left(a^{t} b^{1-t}\right) g\left(a^{t} b^{1-t}\right)+f\left(b^{t} a^{1-t}\right) g\left(b^{t} a^{1-t}\right)\right] \mathrm{d} t \\
= & \frac{1}{\ln b-\ln a} \int_{a}^{b} \frac{f(x) g(x)}{x} \mathrm{~d} x .
\end{aligned}
$$

The proof of Theorem 7 is complete.

Theorem 8. Let $f, g: I \subseteq \mathbb{R}_{+} \rightarrow \mathbb{R}_{+}$be integrable functions, $a, b \in I$ with $a<$ $b$, and $s_{1}, s_{2} \in(0,1]$. If $f$ is an $s_{1}$-geometrically convex function and $g$ is an $s_{2}$ geometrically convex function on $[a, b]$, then

$$
\begin{aligned}
& \sqrt{a b} f^{(1 / 2)^{1-s_{1}}}(\sqrt{a b}) g^{(1 / 2)^{1-s_{2}}}(\sqrt{a b}) \leq \frac{1}{\ln b-\ln a} \int_{a}^{b} f(x) g(x) \mathrm{d} x \\
& \leq[f(a) f(b)]^{1-s_{1}}[g(a) g(b)]^{1-s_{2}} L\left(a f^{s_{1}}(a) g^{s_{2}}(a), b f^{s_{1}}(b) g^{s_{2}}(b)\right),
\end{aligned}
$$

where $L(u, v)$ is the logarithmic mean defined by $(2.1)$.

Proof. Setting $x=a^{t} b^{1-t}$ for $t \in[0,1]$ and making use of Definition 4 arrive at

$$
\begin{aligned}
& \frac{1}{\ln b-\ln a} \int_{a}^{b} f(x) g(x) \mathrm{d} x=\int_{0}^{1} a^{t} b^{1-t} f\left(a^{t} b^{1-t}\right) g\left(a^{t} b^{1-t}\right) \mathrm{d} t \\
& \leq \int_{0}^{1} a^{t} b^{1-t} f^{t^{s_{1}}}(a) f^{(1-t)^{s_{1}}}(b) g^{t^{s_{1}}}(a) g^{(1-t)^{s_{2}}}(b) \mathrm{d} t \\
& \leq[f(a) f(b)]^{1-s_{1}}[g(a) g(b)]^{1-s_{2}} L\left(a f^{s_{1}}(a) g^{s_{2}}(a), b f^{s_{1}}(b) g^{s_{2}}(b)\right)
\end{aligned}
$$


and

$$
\begin{aligned}
& \sqrt{a b} f^{(1 / 2)^{1-s_{1}}}(\sqrt{a b}) g^{(1 / 2)^{1-s_{2}}}(\sqrt{a b}) \\
\leq & \int_{0}^{1}\left(a^{t} b^{1-t} b^{t} a^{1-t}\right)^{1 / 2}\left[f\left(a^{t} b^{1-t}\right) f\left(b^{t} a^{1-t}\right) g\left(a^{t} b^{1-t}\right) g\left(b^{t} a^{1-t}\right)\right]^{1 / 2} \mathrm{~d} t \\
\leq & \frac{1}{2} \int_{0}^{1}\left[a^{t} b^{1-t} f\left(a^{t} b^{1-t}\right) g\left(a^{t} b^{1-t}\right)+b^{t} a^{1-t} f\left(b^{t} a^{1-t}\right) g\left(b^{t} a^{1-t}\right)\right] \mathrm{d} t \\
= & \frac{1}{\ln b-\ln a} \int_{a}^{b} f(x) g(x) \mathrm{d} x .
\end{aligned}
$$

The proof of Theorem 8 is thus complete.

\section{ACKNOWLEDGEMENTS}

This work was partially supported by the National Natural Science Foundation of China (Grant No. 11361038), by the Foundation of Research Program of Science and Technology at Universities of Inner Mongolia Autonomous Region in China (Grant No. NJZZ18154), and by the Science Research Fund of Inner Mongolia University for Nationalities in China (Grant No. NMDGP1714, Grant No. NMDYB1748, and Grant No. NMDGP17104).

\section{REFERENCES}

[1] M. W. Alomari, M. Darus, and U. S. Kirmaci, "Some inequalities of Hermite-Hadamard type for s-convex functions," Acta Math. Sci. Ser. B Engl. Ed., vol. 31, no. 4, pp. 1643-1652, 2011. [Online]. Available: https://doi.org/10.1016/S0252-9602(11)60350-0

[2] R.-F. Bai, F. Qi, and B.-Y. Xi, "Hermite-Hadamard type inequalities for the $m$ - and $(\alpha, m)$-logarithmically convex functions," Filomat, vol. 27, no. 1, pp. 1-7, 2013. [Online]. Available: https://doi.org/10.2298/FIL1301001B

[3] W. W. Breckner, "Stetigkeitsaussagen für eine Klasse verallgemeinerter konvexer Funktionen in topologischen linearen Räumen,” Publ. Inst. Math. (Beograd) (N.S.), vol. 23(37), pp. 13-20, 1978.

[4] S. S. Dragomir and R. P. Agarwal, "Two inequalities for differentiable mappings and applications to special means of real numbers and to trapezoidal formula," Appl. Math. Lett., vol. 11, no. 5, pp. 91-95, 1998. [Online]. Available: https://doi.org/10.1016/S0893-9659(98)00086-X

[5] H. Hudzik and L. Maligranda, "Some remarks on s-convex functions," Aequationes Math., vol. 48, no. 1, pp. 100-111, 1994. [Online]. Available: https://doi.org/10.1007/BF01837981

[6] U. S. Kirmaci, M. Klaričić Bakula, M. E. Özdemir, and J. Pečarić, "Hadamard-type inequalities for s-convex functions," Appl. Math. Comput., vol. 193, no. 1, pp. 26-35, 2007. [Online]. Available: https://doi.org/10.1016/j.amc.2007.03.030

[7] U. S. Kirmaci, "Inequalities for differentiable mappings and applications to special means of real numbers and to midpoint formula," Appl. Math. Comput., vol. 147, no. 1, pp. 137-146, 2004. [Online]. Available: https://doi.org/10.1016/S0096-3003(02)00657-4

[8] B.-Y. Xi, R.-F. Bai, and F. Qi, "Hermite-Hadamard type inequalities for the $m$ - and $(\alpha, m)$-geometrically convex functions," Aequationes Math., vol. 84, no. 3, pp. 261-269, 2012. [Online]. Available: https://doi.org/10.1007/s00010-011-0114-x

[9] B.-Y. Xi and F. Qi, "Some integral inequalities of Hermite-Hadamard type for convex functions with applications to means," J. Funct. Spaces Appl., pp. Art. ID 980 438, 14, 2012. 
[10] B.-Y. Xi and F. Qi, "Some Hermite-Hadamard type inequalities for differentiable convex functions and applications," Hacet. J. Math. Stat., vol. 42, no. 3, pp. 243-257, 2013.

[11] B.-Y. Xi and F. Qi, "Hermite-Hadamard type inequalities for geometrically $r$-convex functions," Studia Sci. Math. Hungar., vol. 51, no. 4, pp. 530-546, 2014. [Online]. Available: https://doi.org/10.1556/SScMath.51.2014.4.1294

[12] B.-Y. Xi and F. Qi, "Inequalities of Hermite-Hadamard type for extended $s$-convex functions and applications to means," J. Nonlinear Convex Anal., vol. 16, no. 5, pp. 873-890, 2015.

[13] B. Y. Xi and F. Qi, "Some integral inequalities of Hermite-Hadamard type for $s$-logarithmically convex functions," Acta Math. Sci. Ser. A Chin. Ed., vol. 35, no. 3, pp. 515-524, 2015.

[14] T.-Y. Zhang, A.-P. Ji, and F. Qi, "On integral inequalities of Hermite-Hadamard type for $s$ geometrically convex functions," Abstr. Appl. Anal., pp. Art. ID 560 586, 14, 2012.

[15] T.-Y. Zhang, M. Tunç, A.-P. Ji, and B.-Y. Xi, "Erratum to "On integral inequalities of Hermite-Hadamard type for $s$-geometrically convex functions" [mr2975277]," Abstr. Appl. Anal., pp. Art. ID 294 739, 5, 2014. [Online]. Available: https://doi.org/10.1155/2014/294739

\section{Authors' addresses}

\section{Hong-Ping Yin}

College of Mathematics, Inner Mongolia University for Nationalities, Tongliao, Inner Mongolia, 028043, China

E-mail address: hongpingyineqq.com

\section{Jing-Yu Wang}

College of Mathematics, Inner Mongolia University for Nationalities, Tongliao, Inner Mongolia, 028043, China

E-mail address: jing-yu.wangeqq.com

\section{Feng Qi}

Corresponding author, Institute of Mathematics, Henan Polytechnic University, Jiaozuo, Henan, 454010, China, Department of Mathematics, College of Science, Tianjin Polytechnic University, Tianjin, 300387, China and College of Institute of Fundamental and Frontier Sciences, University of Electronic Science and Technology of China, Chengdu, Sichuan, 610054, China

E-mail address: qifeng618@gmail.com; qifeng618@hotmail.com 